\title{
ON THE ESTIMATION OF FREE DIPHTHERIA TOXIN :
}

\section{WITH REFERENCE TO THE RELATIONS EXISTING BETWEEN LETHAL DOSES, LETHAL TIMES AND LOSS IN WEIGHT OF THE GUINEA-PIG.}

\author{
By J. A. CRAW, \\ Grocers' Company Research Scholar, \\ Hon. Demonstrator in Physiology, London Hospital Medical College, \\ AND GEORGE DEAN, \\ Chief Bacteriologist Lister Institute, London. \\ (From the Serum Laboratories, Lister Institute.)
}

THE unit lethal dose of a fluid containing free diphtheria toxin may be defined, by Ehrlich's method of standardisation, as the smallest quantity of the fluid which, diluted to 4 c.c. with saline or water and injected subcutaneously into a healthy guinea-pig of 250 to 280 grams weight, causes death in 4 to 5 days. The standardisation of a toxin is usually made in two ways-(1) various dilutions of the toxin are injected and the unit or minimal lethal dose, M.L.D., obtained; (2) various dilutions of the toxin are mixed with an empirically fixed quantity of antitoxin-the Ehrlich immunisation unit, I.E. ${ }^{1-a n d}$ are injected into a series of guinea-pigs. By this test the amount of toxin necessary to neutralise the unit of antitoxin and still leave a lethal dose in excess, usually denoted as the $\mathrm{L}+$ value of the toxin, is determined. When the quantity of toxin is, in both cases, greater than the unit lethal dose the time required for death or lethal time is shortened, and with smaller quantities of toxin death is delayed beyond the standard time. Further, lethal doses usually produce a daily diminution in the weight of the test animal until death ensues, and sublethal doses give rise to a temporary decrease in weight.

1 Immunisierungseinheit. 
In a recent investigation of the neutralisation of diphtheria toxin by antitoxin, Arrhenius and Madsen (1904) have deduced a quantitative relation between lethal dose and lethal time, and likewise an empirical connection between dose of toxin and loss of weight in the test animals. If the observations of Arrhenius and Madsen be found to agree with those on other brews of diphtheria toxin obviously standardisations would be rendered more accurate or a saving in animal life could be effeeted. In their further experiments, designed to test the validity of their views on the toxin-antitoxin reaction, Arrhenius and Madsen find that their observations, interpreted according to the above-mentioned relations, are in close agreement with the theoretically derived values for free diphtheria toxin. Nernst (1904) has pointed out that the equation used by Arrhenius and Madsen is merely an interpolation formula, and the close agreement between calculated and observed results does not prove the validity of their hypothesis. Their observations, however, seem to show that diphtheria toxin is gradually and continuously neutralised by increasing amounts of antitoxin, a result which is in opposition to the experimental investigations and views of Ehrlich (1898), (1903), according to whom the neutralisation takes place in steps owing to the successive combination of several distinct toxic and atoxic substances with different combining affinities present in the toxin. From Arrhenius and Madsen's point of view the step-like neutralisation found by Ehrlich and his pupils is to be interpreted as, in part, due to experimental errors, and the smoothness of the neutralisation curves which they, themselves, have obtained is, in part, due to the more profitable interpretation of their experimental results gained by the application of empirical relations between lethal doses, lethal times, and loss of weight.

It seemed, therefore, to the authors to be important to control the relations deduced by Arrhenius and Madsen, both from the standpoint of toxin and antitoxin standardisation, and the physico-chemical investigation of the toxin-antitoxin reaction by experiments in vivo. Fortunately an extensive record of data had been collected by one of us at the Lister Institute of Preventive Medicine during his standardisation of antitoxic sera, within the period 1900 to 1905 , and these observations have been subjected to detailed analysis by the other. In this communication the chief features of a preliminary examination of some of these observations are presented, pending an investigation by more refined statistical methods, such as those of Prof. Karl Pearson. One of us, viz. G. Dean, is responsible for the experimental data alone, and the other, J. Craw, 


\section{The Estimation of Free Diphtheria Toxin}

for the calculations and conclusions, carried out during his tenure of scholarships from the "Lister Institute," the "British Medical Association" and the "Grocers' Company."

\section{Lethal Doses of Toxin and Time of Death.}

In Table I the relations found by Arrhenius and Madsen to hold between lethal doses and lethal times in days, columns 1 and 2 , are reproduced. In this connection that quantity of toxin which killed with

\section{TABLE I.}

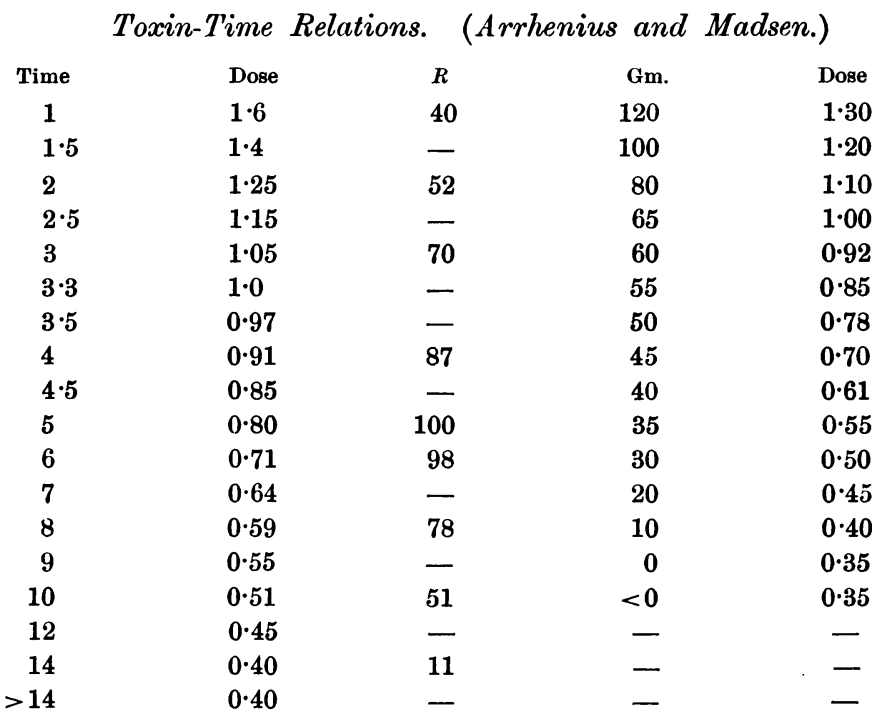

certainty in $3 \cdot 3$ days was taken as unit toxin or minimum lethal dose, M.L.D., but it is of course easy to calculate proportional series of doses when another time limit is selected for the M.L.D., e.g. 3, 4 or 5 days; thus in Table II we give the lethal doses obtained from Arrhenius and Madsen's relation for the second to the sixth day on the basis of a 3-day unit or M.L.D., series "L. dose A. and M." The dotted curve with crosses in Fig. 1 gives a graphical representation of this relation, the lethal doses being shown on ordinates, and the corresponding lethal times on the abscissae. Further, Arrhenius and Madsen assume that if a guineapig recovers, its maximum loss of weight will, in general, occur on the fifth day after inoculation and that the loss in weight on any particular day up to the fourteenth will bear a definite ratio to the maximum. 
Thus, if the maximum loss on the fifth day be taken as 100 the decrement in weight on the first day would have the relative value 40 . This series of ratios is reproduced in Table I, column $3(R)$, and it is possible, by means of it, to calculate from the loss of weight on the first or the second, third, etc. day, what the maximum loss of weight would be on the fifth day, as the absolute loss on any particular day has merely to be multiplied by a definite known ratio. In this manner the loss in weight on each day, both of pigs which survive and of those which die, gives a calculated value for the maximum for which an average can then be obtained. Arrhenius and Madsen consider that a definite relation exists between the maxima so obtained and the dose of toxin injected, and this is reproduced in Table I, columns 4 and 5. The series of weight ratios is represented diagrammatically in Fig. 2, the abscissae giving the time in days and the ordinates the ratio scale 0 to 100 .

TABLE II.

Toxin-Time Relations,

$\begin{array}{lccccc}\text { Lethal time in days } & 2 & 3 & 4 & 5 & 6 \\ \text { Lethal dose } & 1.8 & 1.1 & 0.8 & 0.7 & 0.65 \\ \text { Range of dose } & 5-1.4 & 2-1.0 & 1.2-0.7 & 0.9-0.5 & 0.8-0.45 \\ \text { L. dose (A. \& M.) } & 1.2 & 1.0 & 0.87 & 0.76 & 0.67\end{array}$

The uncertainty associated with the estimation of free diphtheria toxin, owing to variation in the susceptibility of individual guinea-pigs, appears to us to bring these determinations into the class of phenomena which must be treated by more general methods of probability. The deviation from the most probable result decreases as the number of pigs tested increases; thus the problem is to find the likelihood of a result derived from a limited number of tests agreeing approximately with an expected value, provided we proceed on the basis of an hypothesis such as that of Arrhenius and Madsen. If, on the other hand, we proceed according to the method of Ehrlich the probabilities are unknown and we have the inverse problem, viz., to elucidate the M.L.D. and $L+$ values and their theoretical significance from the experimental data. In the present preliminary communication we have adopted the latter method to control the relations between lethal time, dose of toxin and loss of weight. It may, in parenthesis, be remarked that Ehrlich's rectangular method of representation of the neutralisation of toxin by antitoxin would, if carried out systematically, give a better idea of the 
exact value of the experimental data, but that Arrhenius and Madsen's curves give a better idea of the course of the reaction.

In order to control this relation the data referring to 200 different brews of diphtheria toxin were tabulated, and average values were found for the relation between lethal doses and lethal times. Owing to the large number of estimations the arithmetic and geometric means did not differ appreciably.

The range of dose causing death on any particular day is considerable: for the first day it is very great and no upper limit can be given, for the second to the sixth days the ranges are given in Table II, for durations exceeding seven days no relation whatever could be found. In all cases the values given are the arithmetic means. It may be observed that there are more terms ${ }^{1}$ obtainable from any single series of estimations of diphtheria toxin than there are constants, and consequently all the terms must be used in deducing a relation between lethal times and lethal doses. Such a relation has been found for the abovementioned 200 toxins and is given in Table II, Series 1 and 2, the graphical representation of which is to be seen in the continuous curve of Fig. 1. This graduation curve does not reproduce any of the observations exactly, but it runs evenly through the roughness of the observations and so indicates their general trend. These average values are also probably not a true representation of the experimental data, but only an approximation ; it seems to us not improbable that the "mode," i.e. the lethal dose which on a particular day most often causes death, is probably a better guide to the toxin-time relation than the " mean," for owing to the spread or scatter of the observations being, in any selected day, unsymmetrical about the mean and congregating during the first, second and thirds days in the neighbourhood of the longer periods, the frequency curves exhibit a pronounced skewness, a matter which will be dealt with in another paper. This may be gathered from Fig. 1, in which the black circles on the continuous curve represent the means, whereas the straight lines corresponding to each circle represent the ranges-the deviation from symmetry is apparently not a negligible factor.

Omitting for the present the toxin-time relation for the first day, it will be seen, from Table II and Fig. 1, that the lethal dose 1.2 obtained by Arrhenius and Madsen for the second day differs considerably from our values, viz. 1.8, but that on the third day the values correspond

\footnotetext{
1 i.e. lethal values for the series of days considered.
} 
closely, and on the fourth, fifth and sixth days are practically identical. The relation we have obtained seems capable of simpler expression than that of Arrhenius and Madsen, for we have the approximate relation that

Lethal Dose $\times$ Lethal Time $=$ Constant.

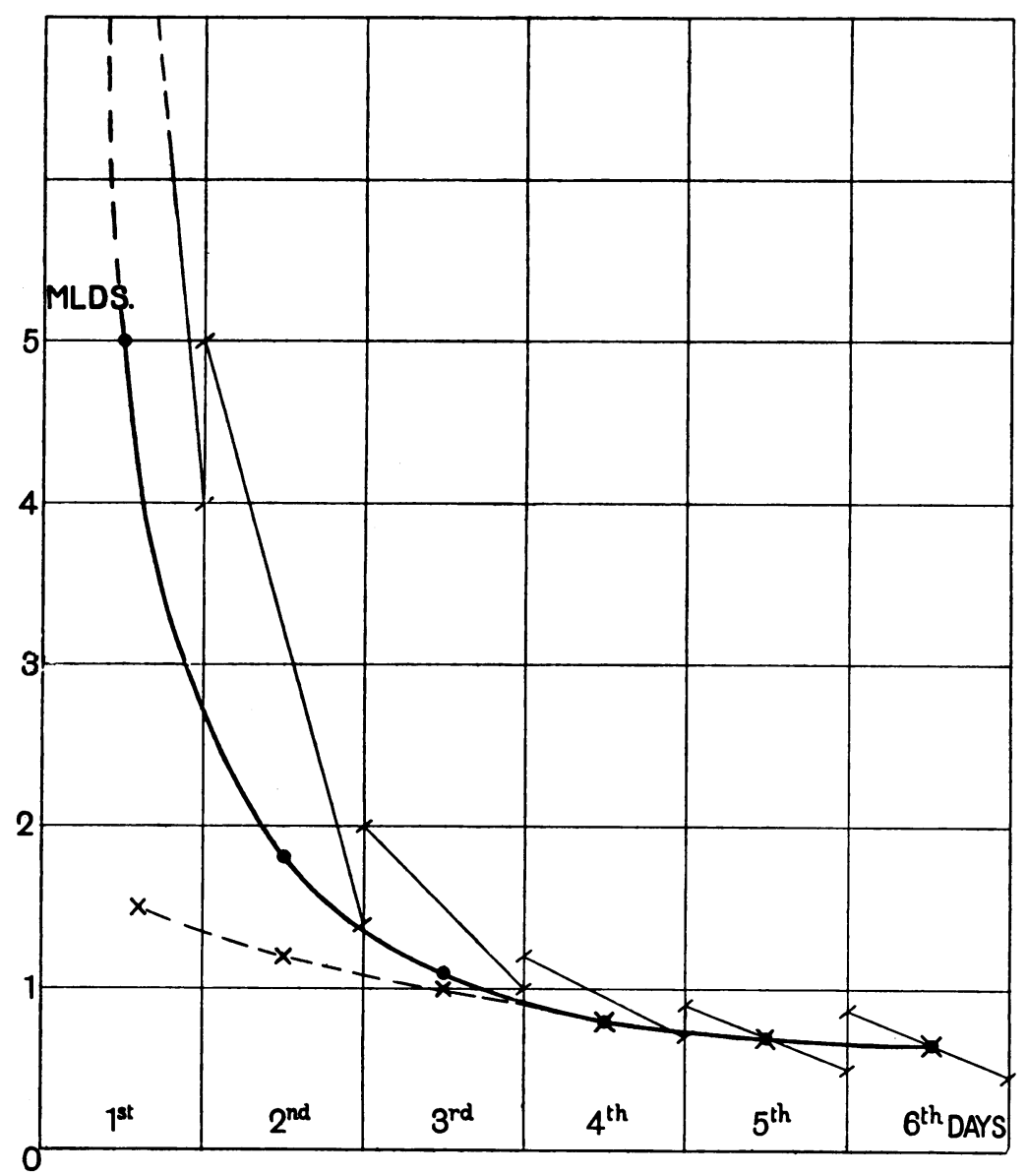

Fig. 1.

Thus the doses killing from the second to the sixth day give the products $3 \cdot 6,3 \cdot 3,3 \cdot 2,3.5$ and 3.9 . The corresponding products from Arrhenius and Madsen's relation are 2.4, 3, 3·48, 3·80, 3.92. The simple relation given, although probably not exactly of the best fitting type, which seems to be Lethal Time $\times \sqrt[n]{\text { Lethal Dose }}=$ Constant, where $n$ is a second constant, is likely to prove serviceable as a rough guide, giving 
results within the experimental error in the customary series of five guinea-pigs used in the standardisation of diphtheria toxins. It is certainly a useful mnemonic, for it states that the time of death is nearly inversely proportional to the dose of toxin. It seems to us to be doubtful whether any closer approximation is attainable under ordinary circumstances as regards the number of guinea-pigs likely to be used in standardisation. This is clearly shown by Table III for Toxin, No. 116, where the number of deaths are given and the days upon which death took place when various amounts of toxin were administered.

TABLE III.

Diphtheria Toxin No. 116 : filtered 28/1/01. Tested 1904.

\begin{tabular}{|c|c|c|c|c|c|c|c|c|c|c|}
\hline \multirow{2}{*}{$\begin{array}{l}\text { Toxin } \\
\text { in c.c. }\end{array}$} & \multirow{2}{*}{$\begin{array}{c}\text { No. of } \\
\text { pigs }\end{array}$} & \multicolumn{8}{|c|}{ Time of death + in days } & \multirow{2}{*}{$\begin{array}{l}\text { No. of } \\
\text { survivals }\end{array}$} \\
\hline & & 1 & 2 & 3 & 4 & 5 & 6 & 7 & tdays & \\
\hline 0.01 & 3 & - & - & tt & $t$ & - & - & - & - & - \\
\hline 0.0075 & 1 & - & + & - & - & - & - & - & - & - \\
\hline 0.005 & 4 & - & $\dagger$ & t† & - & $\dagger$ & - & - & - & - \\
\hline 0.004 & 3 & - & - & $t+t$ & - & - & - & - & - & - \\
\hline 0.0033 & 5 & - & - & - & t+t & - & - & $\dagger$ & +30 & - \\
\hline 0.0029 & 3 & - & - & - & - & - & - & - & +10 & 2 \\
\hline 0.0025 & 3 & - & - & - & - & - & $\dagger$ & - & +24 & 1 \\
\hline
\end{tabular}

It is noteworthy that a definite lethal amount of toxin cannot be relied upon to kill on any particular day, but the probability is that it will kill on one of two days immediately succeeding each other. This table seems to us to be typical of the usual experience met with in the determination of minimal lethal doses, for even in this case, where 22 guinea-pigs were used as test animals, the lethal dose 0.005 c.c. could not be relied upon to kill with absolute certainty on the third day, nor did 0.0033 c.c. give a lethal effect always on the fourth.

Further, the examination of the above-mentioned 200 brews of diphtheria toxin demonstrates conclusively that the number of animals dying on the seventh day to about the twenty-first day is so small and the irregularities so great as to render any calculation of a lethal dose for this period of no practical value.

\section{Lethal Effect of Toxin-Antitoxin Mixtures.}

As the $\mathrm{L}+$ dose of toxin denotes that quantity of toxic fluid which neutralises the unit of antitoxin, I.E., and still gives a minimal lethal dose in excess, it is evident that when the time limit for the $\mathrm{L}+$ dose is varied from the second to the sixth day the relative values will approxi- 
mate more closely than we found to be the case for lethal doses of toxic fluid alone. This can be seen from Tables IV and V. In Table IV the range of $\mathrm{L}+$ dose killing on any day after inoculation is given from the year 1901 to 1905 for the best investigated toxin, viz. No. 116. The average values are shown in the last series, and taking the $\mathrm{L}+$ dose for

TABLE IV ${ }^{1}$

Toxin No. 116 in dilution $1: 5, L+$ in c.c.

\begin{tabular}{|c|c|c|c|c|c|}
\hline \multicolumn{5}{|c|}{ Time of death in days } & \multirow[b]{2}{*}{ Date } \\
\hline 2 & 3 & 4 & 5 & 6 & \\
\hline$\cdot 6$ & $\cdot 61$ & $\cdot 63$ & $\cdot 61$ & $\cdot 60$ & April-Sept. 1901 \\
\hline$\cdot 65$ & .75 & $\cdot 70$ & .62 & - & \\
\hline
\end{tabular}

$\begin{array}{llllll}(4) & (8) & (3) & (3) & (1) & \\ \cdot 72 & \cdot 70 & \cdot 64 & \cdot 74 & \cdot 66 & \text { Oct.-Oct. 1901-2 } \\ \cdot 78 & \cdot 76 & \cdot 70 & - & \cdot 68 & \\ (13) & (14) & (2) & (1) & (2) & \\ \cdot 68 & \cdot 68 & \cdot 68 & \cdot 70 & \cdot 68 & \text { Oct._Oct. 1902-3. } \\ \cdot 78 & \cdot 74 & \cdot 74 & - & - & \\ (14) & (8) & (5) & (1) & (1) & \\ \cdot 70 & \cdot 68 & \cdot 68 & \cdot 66 & \cdot 68 & \text { Oct.-Oct. 1903-4 } \\ \cdot 74 & \cdot 76 & \cdot 72 & \cdot 74 & \cdot 70 & \\ (3) & (10) & (4) & (3) & (3) & \\ \cdot 70 & \cdot 68 & - & \cdot 68 & - & \text { Oct.-Feb. 1904-5 } \\ \cdot 74 & \cdot 74 & - & - & - & \\ (3) & (8) & - & - & - & \\ \cdot 73 & \cdot 72 & \cdot 70 & \cdot 70 & \cdot 68 & 1901-5\end{array}$

TABLE $V$.

$\underset{\text { Lethal time. }}{\text { Days }}$
2
3
4
5
5
6

\begin{tabular}{ccc}
\multicolumn{3}{c}{$L+$ doses corresponding } \\
\hline No. 116 & No. 58 & Mean \\
1.015 & 1.02 & 1.017 \\
1.00 & 1.00 & 1.000 \\
0.97 & 1.00 & 0.985 \\
0.97 & 0.97 & 0.970 \\
0.95 & 0.97 & 0.960
\end{tabular}

the third day as unity the relative values for death on the other days are indicated in Table V. From Table V it may be seen that the range of dose for any particular day is considerable, but the "scatter" of the data is such that the mean values have an experimental error of $\pm 2 \%$ at the outside.

1 Bracketed figures indicate number of test animals. 


\section{The Estimation of Free Diphtheria Toxin}

A very similar relation was obtained for another toxin, No. 58, as is indicated in Table V. Taking the mean of these observations, we find that the $\mathrm{L}+$ doses and corresponding lethal times show a straight line relationship.

It follows also from Table IV that the neutralising power of the toxin No. 116 did not change appreciably during the period October, 1901, to February, 1905, although during this period the minimum lethal dose had increased by $100 \%$, viz. 0.002 c.c. to 0.004 c.c. This is confirmatory of Ehrlich's view that on standing the toxic property of diphtheria toxin diminishes, whereas its neutralising power is, within the experimental error, unchanged. From April, 1904, onwards the toxicity of No. 116 appears to have remained constant, and the curve representing the decrement with time of standing is probably of hyperbolic form and similar to those found by Madsen for the disappearance of antitoxin from the animal system, the deterioration of tetanolysin, etc. A corresponding diminution in toxicity and constancy of neutralising power was observed for toxins Nos. 58, 27, 136 and 198.

The influence of the variation in the susceptibility of the test animals is also markedly shown on injecting mixtures of toxin-antitoxin. Table VI gives a summary of the results obtained when

TABLE VI.

Lethal Effect of Toxin-Antitoxin Mixtures.

Toxin No. 116 in dilution $1: 5+1$ I.E.

\begin{tabular}{|c|c|c|c|c|c|c|c|c|c|}
\hline \multirow{2}{*}{$\begin{array}{l}\text { Toxin } \\
\text { c.c. }\end{array}$} & \multirow{2}{*}{$\begin{array}{l}\text { Pigs } \\
\text { No. }\end{array}$} & \multicolumn{6}{|c|}{ Days } & \multicolumn{2}{|c|}{ Survivors } \\
\hline & & 1 & 2 & 3 & 4 & 5 & 6 & No. & $\%$ \\
\hline $1.2-0.8$ & 10 & 6 & 4 & 0 & 0 & 0 & 0 & 0 & 0 \\
\hline $0.8-0.75$ & 15 & 1 & 9 & 4 & 0 & 0 & 0 & 1 & $6 \cdot 6$ \\
\hline $0.75-0.7$ & 33 & 0 & 10 & 15 & 2 & 1 & 0 & 5 & 15 \\
\hline $0.7-0.65$ & 20 & 0 & 2 & 7 & 3 & 0 & 2 & 6 & 30 \\
\hline $0.65-0.6$ & 32 & 0 & 5 & 4 & 2 & 3 & 1 & 17 & 53 \\
\hline $0.6-0.5$ & 16 & 0 & 1 & 0 & 0 & 0 & 1 & 14 & 87 \\
\hline $0.5-0.4$ & 10 & 0 & 0 & 0 & 0 & 0 & 0 & 10 & 100 \\
\hline $0.4-0.3$ & 8 & 0 & 0 & 0 & 0 & 0 & 0 & 8 & 100 \\
\hline $0.3-0.2$ & 5 & 0 & 0 & 0 & 0 & 0 & 1 & 4 & 80 \\
\hline
\end{tabular}

different amounts of toxin were used, the fluid containing unit antitoxin, i.e. 1 I.E., in each case. For example, with doses between 0.7 and 0.75 c.c. of a 1 in 5 dilution of toxin No. 116, out of 33 guinea-pigs, 10 died on the second day, 15 on the third, 2 on the fourth, 1 on the fifth, 0 on the first and sixth, while 5 pigs, or $15 \%$ of the whole number, survived the sixth day. It seems to be clear from this table, which we 
regard as very typical of the phenomena met with in investigating $\mathrm{L}+$ doses, that the determination of the dose corresponding to any particular lethal time must be the result of the interpolation of a large number of experiments, and that any relation found to exist between lethal dose and lethal time can form, at the best, only a rough guide in the estimation of free toxin in mixtures of toxin and antitoxin.

\section{Loss of Weight and Lethal Time.}

An endeavour was made to control the relations deduced by Arrhenius and Madsen between the variation in weight of the test animals and the time elapsing after inoculation, all the series of Table VI for toxin No. 116 being examined and mean values obtained for loss of weight on any particular day from the first to the fourteenth. The first and most striking result was that, although guinea-pigs which recovered showed at some period a maximum loss of weight, this maximum did not occur for all doses on the fifth day. Further, the shapes of the curves connecting lethal time and loss of weight were not similar to that which represents Arrhenius and Madsen's ratios (see Fig. 2). Quite other relations seem to exist; in the first place, non-lethal doses do not depress the weight of the test animals as a rule on the first day, but the weight continues to rise, and, with the smaller doses, at quite a normal rate, as will be seen from $\dot{a}$ subsequent paper. With larger doses the weight at the end of the first day may be constant, slightly increased or slightly depressed. Only with large lethal doses, in general, is a marked depression obtainable. Secondly, the injection of sub-lethal doses, in which the antitoxin is in great excess, leads in many cases to a marked temporary depression on the first day. This we attribute to "shock," for it has been observed that on the subcutaneous injection of perfectly innocuous fluids, such as normal saline, distilled water, distillates of broth, etc., a similar decrement of weight took place during the 24 hours succeeding inoculation. The mean values of a large number of tests show, however, that the relation first mentioned holds in general, viz., that after the injection of all mixtures of diphtheria toxin and antitoxin the animal continues to increase in weight, but that with increasing amounts of free toxin this increment in weight is more rapidly followed by a decrement, and with non-lethal doses the maximum weight of the pig is found at the end of 24 hours. It appears to us that these maximal weights would, if they were ascertained either by direct determination or by interpolation from a few observations, form 
more reasonable origins for the weight-time curves corresponding to different doses. As an approximation, especially in view of the unreliability of the results obtained during the first 24 hours, we decided to take the weight at the end of the first day as the origin of each curve, or, in other words, as the initial weight of the test animal. On pursuing this course several relations, formerly obscured, became immediately apparent, and these may be gathered from Fig. 2, curves 1 to 6 . For pigs which recovered no maximum loss in weight occurred for doses of 0.4 c.c. to 0.3 c.c. of toxin No. 116 corresponding to the eighth series of Table VI, as shown in Fig. 2, curve 6. Series 7, Table VI, gave curve 5

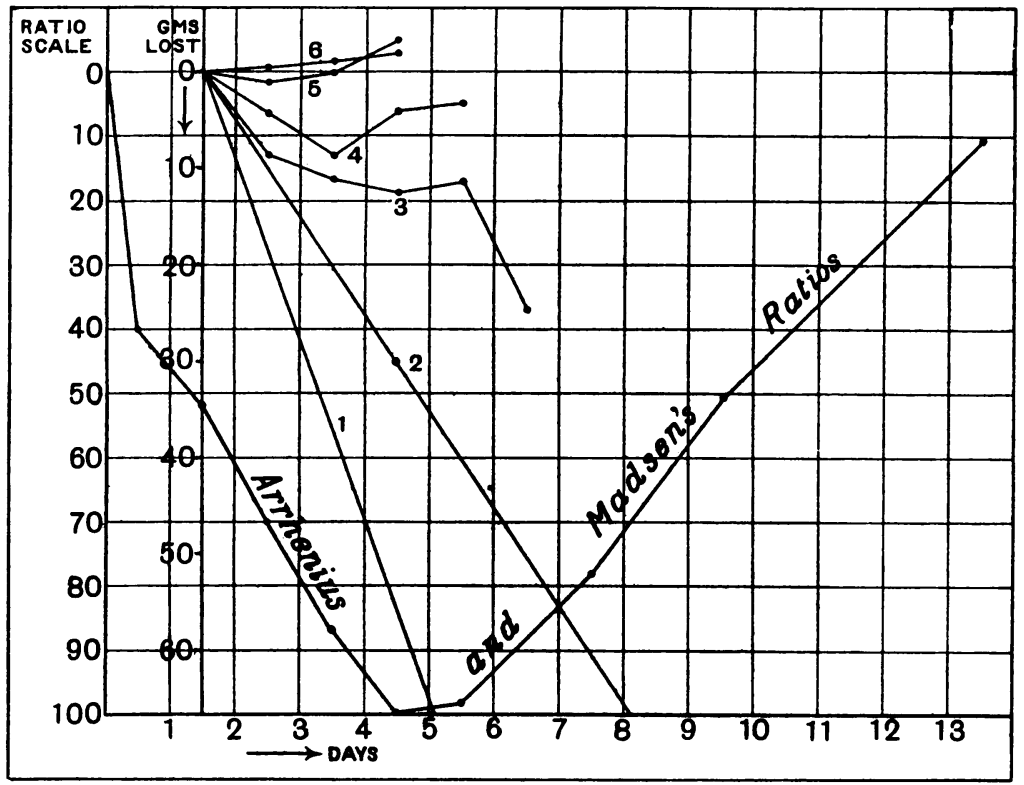

Fig. 2.

in the same figure, and indicates that with 0.5 c.c. to 0.4 c.c. of the diluted toxin the maximum loss of weight took place on the third day. Curve 4, belonging to series 6 , Table VI, gives a maximum loss on the fourth day for 0.6 c.c. to 0.5 c.c. of the toxic fluid. For series 5 no definite maximum loss could be obtained, and, indeed, the form of curve 3 seems to indicate that this is a transition relation between those holding for pigs which recover and others applicable to pigs which die within the first few days. The general downward trend of the curve is probably due to the fact that only $53 \%$ of the test animals survived the sixth day 
on the injection of 06 c.c. to 0.65 c.c. of toxic fluid. The remaining curves, 2 and 1, show in general the time-weight relations existing for amounts of toxin which can be more accurately described as lethal doses or M.L.DS. and L + doses when antitoxin was present, and represent in the latter case the effects of amounts of toxin No. 116 varying from 0.65 c.c. to 0.8 c.c. of the dilution stated in Table VI. In this lethal zone, enclosed by the two curves now under consideration, the injection of 0.65 c.c. gives rise to curve 2 and increasing quantities of toxin give curves which incline more steeply to the abscissa, until finally with 0.8 c.c. the position occupied by curve 1 is reached.

With doses greater than 0.8 c.c. up to 1.2 c.c. the inclination does not appreciably exceed that of curve 1 , and as the values obtained from these doses refer chiefly to pigs which died within 24 hours they have been omitted. The lethal rays thus obtained for the time-weight relations of doses between 0.65 c.c. and 0.8 c.c. are, within the experimental error, straight line relations, and it will be seen from a subsequent paper that they practically correspond to the "starvation curve" of the guinea-pig under normal conditions.

\section{Summary of Conclusions.}

1. The relation between lethal doses of diphtheria toxin and the times in which they kill guinea-pigs, or lethal times, is approximately such that the lethal dose multiplied by the corresponding lethal time gives a constant value-a hyperbolic relation.

2. Deductions with regard to lethal doses from deaths occurring on the first day or on any day after the sixth give such widely divergent results that, in our opinion, they are at present of negligible value.

3. Under the ordinary circumstances of standardisation of diphtheria toxin and antitoxin the relation given in conclusion No. 1 is as close an approximation as any alternative likely to prove of practical utility.

4. The graphic representation of $\mathrm{L}+$ doses against lethal times gives a straight line relationship.

5. Five of the best investigated toxins confirmed Ehrlich's views in so far as that with toxicities diminishing by $50 \%$ their neutralising powers remained practically unimpaired.

6. The individual sensitivities of guinea-pigs to free diphtheria toxin render any general relation between lethal times and doses of little value when a small number, e.g. 5 , of test animals is inoculated. 


\section{The Estimation of Free Diphtheria Toxin}

7. Even with a dose of toxin which would, in any ordinary series of tests, be regarded as an $\mathrm{L}+$ dose, 5 out of 33 guinea-pigs survived the sixth day or $15 \%{ }^{1}$, and the M.L.D. of a well-investigated toxin showed similar variations; consequently both the $\mathrm{L}+$ and M.L.D. values of a toxin are not those amounts which kill with certainty in a fixed time, but those which will cause death in that time with the greatest probability.

8. After inoculation, guinea-pigs unless suffering from the consequent "shock," continue to increase in weight throughout the first day, even with doses that may subsequently prove lethal, and with highly toxic doses the variation in weight during this time is of little significance.

9. The greater the amount of free toxin injected, the more rapid is the increment in weight followed by a decrement.

10. The weight of a test animal at the end of 24 hours after inoculation forms a better normal or origin with which to compare the subsequent time-weight relations than the initial weight before injection.

11. On the basis of Conclusion 10 the general connection is found that with increasing amounts of free toxin the position of the maximum loss of weight is gradually transferred from the second to the fifth day for guinea-pigs which survive over six days.

12. The time-weight ratios for almost certainly lethal doses give straight line connections, corresponding probably to starvation curves of the guinea-pig.

\section{REFERENCES.}

Arrhenius and Madsen (1904), Travail de l'Institut Sérothérapique de l'état Danois, p. 269.

ERHLICH (1898), Deutsche med. Wochenschr., vol. xxiv. p. 597.

- (1903), Berliner klin. Wochenschr., vol. xL. pp. 793, 825, 848.

Nernst (1904), Zeitschr. f. Electrochemie, vol. x. p. 377.

1 In the standardisation of diphtheria antitoxin at the Lister Institute, Elstree, 0.72 c.c. of toxin No. 116 was considered to represent the $L+$ dose for the fourth or fifth day instead of the third, which, according to the above calculations, would harmonise best with the mean of all the observations. The fluids so standardised had then at least the value ascribed to them and were probably higher in content of antitoxin. 